\title{
Detection with Spatial and Temporal Optimization of Search Efforts Involving multiple Modes and multiple Resources Management
}

\author{
Frédéric Dambreville \\ IRISA/CNRS, \\ Campus de Beaulieu, \\ 35042 Rennes Cedex, France. \\ fdambrev@irisa.fr
}

\author{
Jean-Pierre Le Cadre \\ IRISA/CNRS, \\ Campus de Beaulieu, \\ 35042 Rennes Cedex, France. \\ lecadre@irisa.fr
}

\begin{abstract}
This paper deals with optimization of splitable resources aimed to the detection of a moving target following a Markovian movement or a conditionally deterministic motion. Our work extends Brown's spatial optimization method. By use of a generalized linear formalism, we developed a method for optimizing both spatially and temporally (modeling resource renew), with management of multiple resource types or multi-modes resources. Such optimization involves also the fusion of several detection tools, in order to make them work together efficiently.
\end{abstract}

Keywords: Search theory, Sensor management, resource allocation, Optimization, Detection.

\section{Introduction}

The present work refers to the initial framework of Search Theory [8][1][2] introduced by B.O. Koopman and his colleagues. Our interest more precisely focuses on the multi-period search problem, as it has been solved by S.S. Brown [5][6]. After a short review of the formalism, we will eventually explain in this introduction the main aspects of the algorithm evolved by Brown. Brown's algorithm only solves the spatial sharing of the resources. Section 2 and 3 will present extensions of the common formalism and of the algorithm of Brown. Those extensions concern temporal optimization and multi-resource (or mode) scheduling. Section 4 presents some numerical results.

Definitions: A target moving in a search space $E$ is to be detected. The detection is achieved during $T$ periods, each period being brief enough to satisfy the stationarity hypothesis of the target for the period. The trajectory $\vec{x}=\left(x_{1}, \ldots, x_{T}\right) \in E^{T}$ of the target is described by a probabilistic prior about $\vec{x}$, given by a probabilistic density $\alpha(\vec{x})$. A Markovian assumption will be made for algorithmic reasons; $\alpha$ is taken as a product of elementary densities:

$$
\alpha(\vec{x})=\alpha_{1,2}\left(x_{1}, x_{2}\right) \times \cdots \times \alpha_{T-1, T}\left(x_{T-1}, x_{T}\right) .
$$

A given amount of search effort $\phi_{k}$ is available at each period $k$. Those efforts are constants of the problem. At each period $k$, the effort $\phi_{k}$ may be distributed along $E$ to accomplish the search. The local search effort, applied to the point $x_{k} \in E$ at time $k$, is denoted $\varphi_{k}\left(x_{k}\right)$. Constraints (2) are considered:

$$
\forall k \in\{1, \ldots, T\}, \varphi_{k} \geq 0 \text { and } \int_{E} \varphi_{k}(x) d x=\phi_{k} .
$$

The local efforts $\varphi$ directly command the local detection probability. We call $p_{k, x_{k}}\left(\varphi_{k}\left(x_{k}\right)\right)$ the probability not to detect within the period $k$, when target's location is $x_{k}$. The law of diminishing return is assumed. Thus for $x_{k}$ fixed, $p_{k, x_{k}}^{\prime}<0$ and $p_{k, x_{k}}^{\prime \prime}>0$. The problem is to find $\varphi$ so as to minimize the global probability of non detection $\mathbf{P}_{n d}(\varphi)$ under the constraint (2). Independence hypothesis on detections yields:

$$
\mathbf{P}_{n d}(\varphi)=\int_{E^{T}} \alpha(\vec{x}) \prod_{k=1}^{k=T} p_{k, x_{k}}\left(\varphi_{k}\left(x_{k}\right)\right) \prod_{k=1}^{k=T} d x_{k}
$$

Brown's method: Brown obtained a convergence toward the optimal solution, by successively optimizing alone each period with the other fixed. More precisely, for a particular period $\kappa, \mathbf{P}_{n d}(\varphi)$ can also be written:

$$
\begin{aligned}
& \mathbf{P}_{n d}(\varphi)=\int_{E} \beta_{\kappa}^{\varphi}\left(x_{\kappa}\right) p_{\kappa, x_{\kappa}}\left(\varphi_{\kappa}\left(x_{\kappa}\right)\right) d x_{\kappa}, \text { where: } \\
& \beta_{\kappa}^{\varphi}\left(x_{\kappa}\right)=\int_{E^{T-1}} \alpha(\vec{x}) \prod_{1 \leq k \leq T}^{k \neq \kappa}\left(p_{k, x_{k}}\left(\varphi_{k}\left(x_{k}\right)\right) d x_{k}\right) .
\end{aligned}
$$

This shows that, when the search efforts are fixed for all periods, except for a period $\kappa$, the optimization problem becomes the following 1-period problem:

$$
\begin{gathered}
\text { Minimize } P_{n d}\left(\varphi_{\kappa}\right)=\int_{E} \beta_{\kappa}^{\psi}(x) p_{\kappa, x}\left(\varphi_{\kappa}(x)\right) d x, \\
\text { subject to } \int_{E} \varphi_{\kappa}(x) d x=\phi_{\kappa} \text { and } \varphi_{\kappa} \geq 0 .
\end{gathered}
$$

The following optimality conditions scaled by parameter $\eta$ (equations of de Guenin [4]) are obtained:

$$
\left\{\begin{array}{l}
\beta_{\kappa}^{\varphi}(x) p_{\kappa, x}^{\prime}\left(\varphi_{\kappa}(x)\right)=\eta_{\kappa}, \text { if } \beta_{\kappa}^{\varphi}(x)>\eta_{\kappa} / p_{\kappa, x}^{\prime}(0) \\
\varphi_{\kappa}(x)=0, \text { else }
\end{array}\right.
$$


Since $p_{k, x}^{\prime}$ is strictly monotonic, the equations of de Guenin are invertible. By inverting those equations (while $\eta$ is not specified), an uniformly increasing function $\varphi_{k}^{\eta}$ is obtained. The increaseness of $\varphi_{k}^{\eta}$ makes $\int_{E} \varphi_{\kappa}^{n}(x) d x$ increasing. The good value for $\eta_{\kappa}$ is then obtained by a dychotomic process on $\varphi_{r}^{\eta}$. The whole process requires only a few iterations. It uses basically the Markovian assumption relative to $\alpha$, so as to drastically reduce the computation requirements for the integral (4), (Forward And Backward algorithm [5][6]). This idea will be instrumental again in our algorithm.

\section{Generalized models}

Brown's algorithm assumes $\phi$ to be known and deal$s$ only about resources spatial sharing. Optimization on $\phi_{k}$ is however a worthwhile issue. Time splitting of a global amount of non renewable resources, or allocation problems on renewable resources are examples. A more general problem, called lateral splitting, will be presented. This problem intends to involve multi-resource and multi-mode scheduling together with spatio-temporal optimization of resources. To do that, it combines spatial optimizations (as usually) and global optimizations, which act at the index level (some indexes may not be temporal). Before defining a formalism for lateral splitting, some examples are given. They should enlighten on how lateral splitting constitutes a common canvas for modeling both the nature of particular resources and the combinations of different resources. For our examples, we will restrict to a search on $T=4$ periods.

Modeling resources: A resource is certainly characterized by its non detection functions $p$, but it is not sufficient. The relations between global resources $\phi_{k}$ have to be set. In Brown's formalism, this setting is given only by the constant vector $\phi$. It is a poor formalism, since constantness forbids interaction between periods. For example, if we want to define a non renewable resource, which total amount, say 100 , has to be shared between the 4 periods, it is necessary to handle $\phi$ as a variable and not as a constant. Nevertheless, since resources are non renewable and limited by 100 , variables $\phi$ obey to the 4 constraints:

$$
\left\{\begin{array}{l}
\phi_{1} \leq 100 \\
\phi_{1}+\phi_{2} \leq 100 \\
\phi_{1}+\phi_{2}+\phi_{3} \leq 100 \\
\phi_{1}+\phi_{2}+\phi_{3}+\phi_{4} \leq 100
\end{array}\right.
$$

which rewrite in a matrix formalism:

$$
A \phi \leq \psi \text { with } A=\left(\begin{array}{llll}
1 & 0 & 0 & 0 \\
1 & 1 & 0 & 0 \\
1 & 1 & 1 & 0 \\
1 & 1 & 1 & 1
\end{array}\right) \text { and } \psi=\left(\begin{array}{l}
100 \\
100 \\
100 \\
100
\end{array}\right)
$$

It is noteworthy that, since $\phi \geq 0, \phi$ is sufficiently characterized by constraint $\phi_{1}+\phi_{2}+\phi_{3}+\phi_{4} \leq 100$. Nevertheless, in such form the information on $\phi$ is hidden. We say then, that the constraint is informationally incomplete. Such form will not be compatible (formally and semantically) with our modeling of multi-mode scheduling. Thus, we will never use them.

It is just as easy to define resource renewable after 2 periods. Assume total amount be 50 . Then, it is not difficult to see that variables $\phi$ obey to constraints $\phi_{1} \leq 50, \phi_{1}+\phi_{2} \leq 50, \phi_{2}+\phi_{3} \leq 50$ and $\phi_{3}+\phi_{4} \leq 50$. All is resumed again in a matrix formalism:

$$
A \phi \leq \psi \text { with } A=\left(\begin{array}{llll}
1 & 0 & 0 & 0 \\
1 & 1 & 0 & 0 \\
0 & 1 & 1 & 0 \\
0 & 0 & 1 & 1
\end{array}\right) \text { and } \psi=\left(\begin{array}{l}
50 \\
50 \\
50 \\
50
\end{array}\right)
$$

Combining resources: The two precedent examples will be now indexed so as to distinguish them The non renewable resource is indexed $R \infty$ (i.e. $\phi^{R \infty}$, $A^{R \infty}, \ldots$ ) and the other $R 2$ (i.e. $\phi^{R 2}, A^{R 2}, \ldots$ ). Suppose now, that these two kinds of resources are used simultaneously. The probability $\mathbf{P}_{n d}(\varphi)$ becomes:

$\mathbf{P}_{n d}=\int_{\vec{x} \in E^{T}} \alpha(\vec{x}) \prod_{k=1}^{k=T} p_{k, x_{k}}^{R \infty}\left(\varphi_{k}^{R \infty}\left(x_{k}\right)\right) p_{k, x_{k}}^{R 2}\left(\varphi_{k}^{R 2}\left(x_{k}\right)\right)$

Moreover, since resources are parallelized, constraints on $\phi^{R \infty}$ and $\phi^{R 2}$ are simply keep unchanged and put together. This operation, generalized in next section as operator $\otimes$, yields the following constraint:

$$
\left(\begin{array}{cc}
A^{R \infty} & 0 \\
0 & A^{R 2}
\end{array}\right)\left(\begin{array}{c}
\phi^{R \infty} \\
\phi^{R 2}
\end{array}\right) \leq\left(\begin{array}{c}
\psi^{R \infty} \\
\psi^{R 2}
\end{array}\right)
$$

Now, assume that resources work in two modes, i.e. exactly like resources $R \infty$ in mode 1 and exactly like resources $R 2$ in mode 2 . Expression of probability $P_{n d}$ remains unchanged. Assume also that running mode can be changed only when resources are unused or renewed, and that 100 resources $R \infty$ in mode 1 is equivalent to 50 resources $R 2$ in mode 2 . Then, variables $\phi^{R \infty}$ and $\phi^{R 2}$ obey to new constraints $\phi_{1}^{R \infty}+2 \phi_{1}^{R 2} \leq 100$ and $\sum_{k=1}^{\kappa} \phi_{k}^{R \infty}+2 \phi_{k-1}^{R 2}+2 \phi_{k}^{R 2} \leq 100$, for $\kappa=2,3,4$. This operation, generalized in next section as operator $\oplus$, yields the following constraint:

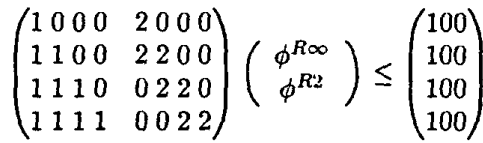

The examples rely on similar formalism. These formalism are unified into lateral splitting, explained now.

\subsection{Formalism for lateral splitting}

Let $T=\{1, \ldots, T\}$ be a set of temporal indices, and $\mathrm{s} \notin \mathcal{T}$ a generic non temporal index. A Lateral Splitting, $\mathbf{L S}$, is a quintuplet $A=(U, \tau, p, \mathcal{A}, m)$, where:

- $U$ is a set of variables indices not contening 1 . Index 1 will be used for indexing constant data,

- $\tau: U \mapsto \mathcal{T} \cup\{\mathrm{S}\}$ is a mapping,

- $p=\left(p_{\Upsilon}\right)_{\Upsilon \in \tau^{-1}(\mathcal{T})}$ are non detection functions verifying $p_{\Upsilon, x}^{\prime}<0$ and $p_{\Upsilon, x}^{\prime \prime}>0$ (diminishing return), 
- $\mathcal{A} \subset \mathbb{R}^{E \cup\{1\}}$ is a set of weighting for variables and constant. Constraints are defined by this object. Note that $\mathcal{A}$ is a kind of non ordered matrix,

- $m: \mathcal{A} \rightarrow \mathcal{P}(\mathcal{T} \cup\{\mathrm{S}\})^{*}$ is a mapping from $\mathcal{A}$, which defines the temporal context of each constraint.

In addition, are defined $\phi=\left(\phi_{\Upsilon}\right)_{\Upsilon \in U}$, a family of global effort variables and $\varphi=\left(\varphi_{\Upsilon}\right)_{\Upsilon \in T^{-1}(\tau)}$, a family of spatial sharing functions. Concretely, $\tau(\Upsilon)=k \in \mathcal{T}$ means that resources $\phi_{\Upsilon}$ are used for search at period $k$. Otherwise, when $\tau(\Upsilon)=\mathrm{S}$, resources $\phi_{\Upsilon}$ are not used. Constraints (7) condition the problem:

$$
\left\{\begin{array}{l}
\forall \Upsilon \in U, \phi_{\Upsilon} \geq 0 \text { and } \forall \Upsilon \in \tau^{-1}(\mathcal{T}), \varphi_{\Upsilon} \geq 0, \\
\forall \Upsilon \in \tau^{-1}(\mathcal{T}), \int_{E} \varphi_{\Upsilon}(x) d x=\phi_{\Upsilon},
\end{array}\right.
$$

Now, in addition to theses basic constraints ( 7$)$, other equation on global variables $\phi_{\Upsilon}$ are evolved from $\mathcal{A}$ :

$$
\forall a \in \mathcal{A}, \sum_{\Upsilon \in U} a(\Upsilon) \phi_{\Upsilon}+a(1) \leq 0 .
$$

The precedent equation has of course a matricial form:

$$
A \phi \leq \psi,
$$

where constraint vector $\psi=(-a(1))_{a \in \mathcal{A}}$ and constraint matrix $A=(a(\Upsilon))_{\Upsilon \in U, a \in \mathcal{A}}$ are constants of the problem. In such model, it appears that the sign of $a(\Upsilon)$ (or $a(1))$ determines whether resources are consumed, positive case, or generated, negative case. The constraints of the problem are now defined. Equation (10) gives the evaluation function to be optimized:

$$
\mathbf{P}_{n d}(\varphi)=\int_{E^{T}} \alpha(\vec{x}) \prod_{k=1}^{k=T}\left(\prod_{\Upsilon \in T^{-1}(k)} p_{\Upsilon, x_{k}}\left(\varphi_{\Upsilon}\left(x_{k}\right)\right)\right) d x_{k}
$$

$\mathbf{P}_{n d}(\varphi)$ represents the global probability of non detection. LS-problem with inequality constraints, ICLS, consists in optimizing both $\phi$ and $\varphi$ so as to minimize $P_{n d}(\varphi)$ under constraints (7) and (8-9).

Equality constraint: LS-problem with equality constraints, ECLS, is just obtained by replacing (8) or (9) by either $\forall a \in \mathcal{A}, \sum_{\Upsilon \in U} a(\Upsilon) \phi_{\Upsilon}+a(1)=0$ or $A \phi=\psi$. ECLS is too restrictive and is inadequate with the underlying semantic of our model. All coming formalism will thus rather refer to ICLS. Nevertheless, our algorithm is built for ECLS, and ICLS is defined as a corollary of ECLS by simply adding slack variables (mapping to S). More precisely, we will add as much new slack variables as cardinality of $\mathcal{A}$. One variable only is activated (weighted with non null value) for each $a \in \mathcal{A}$. Since there is a positivity constraints on variables, it suffices to put a positive weight, say 1 , on needed variable, to handle lower inequality. ICLS $(U, \tau, p, \mathcal{A}, m)$ is simply changed into an equivalent $\mathrm{E}-$ $\operatorname{CLS}\left(U_{\leq}, \tau_{\leq}, p, \mathcal{A}_{\leq}, m_{\leq}\right)$, in the following way:

$$
\left\{\begin{array}{l}
\left.U_{\leq}=U \sqcup \mathcal{A} \text { (disjoint union: } U \cap \mathcal{A}=\emptyset\right), \\
\forall \Upsilon \in U, \tau_{\leq}(\Upsilon)=\tau(\Upsilon) \text { and } \tau_{\leq}(\mathcal{A})=\mathrm{S}
\end{array}\right.
$$

Define $a_{\leq}$for each $a \in \mathcal{A}$, by completion outside $\mathcal{A}$ :

$$
\left\{\begin{array}{l}
\forall \Upsilon \in U \cup\{1\}, a_{\leq}(\Upsilon)=a(\Upsilon), \\
a_{\leq}(a)=1 \text { and } \forall \Upsilon \in \mathcal{A} \backslash\{a\}, a_{\leq}(\Upsilon)=0 .
\end{array}\right.
$$

Objects $\mathcal{A}_{\leq}$and $m_{\leq}$are then defined by:

$$
\left\{\begin{array}{l}
\mathcal{A}_{\leq}=\left\{a_{\leq} / a \in \mathcal{A}\right\}, \\
\forall a_{\leq} \in A_{\leq}, m_{\leq}\left(a_{\leq}\right)=m(a) .
\end{array}\right.
$$

Basic LS: Let $\Lambda=(U, \tau, p, \mathcal{A}, m)$ be an LS. In L$S$ formalism, each index $\Upsilon \in U$ represents a resource variable since index 1 represents the resource constan$t$. These resources are weighted for each constraint $a \in \mathcal{A}$. Weighting signifies that resources may be less or more costly. A resource weighted 2 is equivalent to two resources weighted 1 . A negative weight signifies a reversed cost. It may be used to handle resource generation, and also conditionallity on resources. For example, to explain that a total of $\lambda$ resources is made available by the system, we should use a resource constant weighted by $-\lambda$. Here comes a distinction between resources availability, which depends on the system and is associated to constant index, and resource properties characterized by constraints on variable indices. For this reason, some operations on resources explained next, will be applied rather on LSs with zero weighted constants. Such LS, verifying also $\forall a \in \mathcal{A}, a(1)=0$, are called linear LS. Useful linear LSs will be constructed soon. Otherwise, pure constant LS may be defined and are used together with linear LS. For $\lambda \in \mathbb{R}$, a LS also named $\lambda$ is defined by the quintuplet:

$$
\lambda \triangleq(\emptyset, \emptyset, \emptyset,\{a\}, m), \text { with } a(1)=\lambda, m(a)=\mathcal{T} .
$$

LS for renewable resources: Given a resource type capable of renew after $\Delta T \in \mathbb{N}^{*} \cup\{\infty\}$ periods (time for replenishment, for moving, ...). Consider $\Lambda^{R \Delta T}=\left(U^{R \Delta T}, \tau^{R \Delta T}, p^{R \Delta T}, \mathcal{A}^{R \Delta T}, m^{R \Delta T}\right)$, the linear LS associated. Search is assumed to be done on $T$ periods, so that $\mathcal{T}=\{1, \ldots, T\} . p^{R \Delta T}$ is supposed known. Since the resource is simple (one mode and one type), $\tau^{R \Delta T}$ is one to one. Thus, $U^{R \Delta T}=\left\{\Upsilon_{k} / k \in \mathcal{T}\right\}$ with $\tau^{R \Delta T}\left(\Upsilon_{k}\right)=k$. For same reason, there is a one to one correspondence between constraints and periods. Thus, $\mathcal{A}^{R \Delta T}=\left\{a_{k} / k \in \mathcal{T}\right\}$ and $m^{R \Delta T}\left(a_{k}\right)=\{k\}$. It remains to define the constraint $a_{k}$ associated to the period $k$. It results from the resource balance at period $k$ and is deduced recursively. For period 1, only the cost associated to $\phi_{1}$ are in turn. For period $k$, the cost of $\phi_{k}$ and the possible negative cost (since renew) of $\phi_{k-\Delta T}$ are added to the statement of period $k-1$ :

$$
\left\{\begin{array}{l}
a_{1}\left(\Upsilon_{1}\right)=1 \text { and } \forall k>1, a_{k}\left(\Upsilon_{k}\right)=a_{k-1}\left(\Upsilon_{k}\right)+1, \\
\forall k>\Delta T, a_{k}\left(\Upsilon_{k-\Delta T}\right)=a_{k-1}\left(\Upsilon_{k-\Delta T}\right)-1, \\
a_{1}(\Upsilon)=0 \text { and } \forall k>1, a_{k}(\Upsilon)=a_{k-1}(\Upsilon) \text { else. }
\end{array}\right.
$$

For example, $\mathcal{A}^{R 3}$ yields for $T=4$ the matricial form:

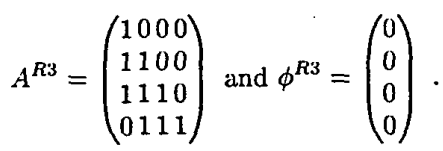


Weighted renewable resources: Resource type is considered, which renews gradually according to a parameter sequence $\rho$. More precisely, for one resource used at period $k, \rho_{\Delta k}$ resource is then regenerated at period $k+\Delta k$. Property $\sum \rho_{k} \leq 1$ also holds. Denote $\Lambda^{R}(\rho)=\left(U_{\rho}^{R}, \tau_{\rho}^{R}, p_{\rho}^{R}, \mathcal{A}_{\rho}^{R}, m_{\rho}^{R}\right)$ the linear LS associated. As previously, there is a one to one correspondence between variables, periods and constraints. We can write $U_{\rho}^{R}=\left\{\Upsilon_{k} / k \in \mathcal{T}\right\}, \mathcal{A}_{\rho}^{R}=\left\{a_{k} / k \in \mathcal{T}\right\}$, with $\tau_{\rho}^{R}\left(\Upsilon_{k}\right)=k$ and $m_{\rho}^{R}\left(a_{k}\right)=\{k\}$. Then, $a_{k}$ is defined by:

$$
\left\{\begin{array}{l}
a_{1}\left(\Upsilon_{1}\right)=1 \text { and } \forall k>1, a_{k}\left(\Upsilon_{k}\right)=a_{k-1}\left(\Upsilon_{k}\right)+1, \\
\forall 1 \leq l<k, a_{k}\left(\Upsilon_{l}\right)=a_{k-1}\left(\Upsilon_{l}\right)-\rho_{k-l}, \\
a_{1}(\Upsilon)=0 \text { and } \forall k>1, a_{k}(\Upsilon)=a_{k-1}(\Upsilon) \text { else. }
\end{array}\right.
$$

For example, $\mathcal{A}^{R}\left(\frac{1}{3}, \frac{1}{2}\right)$ yields for $T=4$ the form:

$$
A^{R}\left(\frac{1}{3}, \frac{1}{2}\right)=\left(\begin{array}{llll}
1 & 0 & 0 & 0 \\
\frac{2}{3} & 1 & 0 & 0 \\
\frac{1}{6} & \frac{2}{3} & 1 & 0 \\
\frac{1}{6} & \frac{1}{6} & \frac{2}{3} & 1
\end{array}\right) \text { and } \phi^{R}\left(\frac{1}{3}, \frac{1}{2}\right)=\left(\begin{array}{l}
0 \\
0 \\
0 \\
0
\end{array}\right) \text {. }
$$

Obviously, there is some semantics, behind our model There is a temporal semantic, which underlies the recursive construction of the LS seen above. It is needless to develop anymore argumentation about it. But it is important to respect the temporal causality when constructing LS. This prevents from irrelevant use of the model, such as informational incompleteness. There is also a global semantic, which establish relation between already constructed LS. This aspect will be more detailed, with the construction of operators on LS.

Global operators: Let $\lambda, \mu \in \mathbb{R}$. For $\alpha=a, b, c$ notation $\Lambda^{\alpha}=\left(U^{\alpha}, \tau^{\alpha}, p^{\alpha}, \mathcal{A}^{\alpha}, m^{\alpha}\right)$ is used. Indices between two LSs $a$ and $b$ are assumed compatible, that is $\Upsilon \in U^{a} \cap U^{b} \Rightarrow \tau^{a}(\Upsilon)=\tau^{b}(\Upsilon), p^{a}(\Upsilon)=p^{b}(\Upsilon)$.

Scalar product operator: The scalar product $\lambda \Lambda^{a}$ is given by the quintuplet $\left(U^{a}, \tau^{a}, p^{a}, \lambda \mathcal{A}^{a}, m^{a}\right)$ where $\lambda \mathcal{A}^{a}=\left\{\lambda a / a \in \mathcal{A}^{a}\right\}$.

Operator $\otimes: \Lambda^{a} \otimes \Lambda^{b}$ is defined as a quintuplet $\left(U^{a} \cup U^{b}, \tau^{a} \cup \tau^{b}, p^{a} \cup p^{b}, \mathcal{A}^{a} \cup \mathcal{A}^{b}, m^{a} \otimes m^{b}\right)$. Function $\tau^{a} \cup \tau^{b}$ is defined by $\forall \Upsilon \in U^{\alpha}, \tau^{a} \cup \tau^{b}(\Upsilon)=\tau^{\alpha}(\Upsilon)$ for $\alpha=a, b$. Similar definition holds for $p^{a} \cup p^{b}$. Now, $m^{a} \otimes m^{b}$ is given by the union of the images:

$$
m^{a} \otimes m^{b}(a)=\bigcup_{A^{\alpha} \ni a} m^{\alpha}(a) .
$$

Operator $(1): \Lambda^{a}(\emptyset) \Lambda^{b}$ is defined as a quintuplet $\left(U^{a} \cup U^{b}, \tau^{a} \cup \tau^{b}, p^{a} \cup p^{b}, \mathcal{A}^{a} \oplus \mathcal{A}^{b}, m^{a}\left(\ni m^{b}\right)\right.$. For all $\left(a^{a}, a^{b}\right) \in \mathcal{A}^{a} \times \mathcal{A}^{b}$, let $a^{a} \oplus a^{b}$ be stated by:

$$
\left\{\begin{array}{l}
\forall \Upsilon \in U^{a} \cup U^{b}, a^{a} \oplus a^{b}(\Upsilon)=\sum_{U^{\alpha} \ni \Upsilon} a^{\alpha}(\Upsilon), \\
a^{a}\left(a^{b}(1)=a^{a}(1)+a^{b}(1) .\right.
\end{array}\right.
$$

$\mathcal{A}^{a}\left(\oplus \mathcal{A}^{b}\right.$ and $m^{a} \oplus m^{b}$ are minimal object defined by:

$$
\left\{\begin{array}{l}
k \in \mathcal{T} \cap m\left(a^{a}\right) \cap m\left(a^{b}\right) \Rightarrow\left\{\begin{array}{l}
a^{a}(1) a^{b} \in \mathcal{A}^{a}(1) \mathcal{A}^{b}, \\
k \in m^{a}(1) m^{b}\left(a^{a}\left(\ominus a^{b}\right),\right.
\end{array}\right. \\
s \in m^{\alpha}\left(a^{\alpha}\right) \Rightarrow\left\{\begin{array}{l}
a^{\alpha} \in \mathcal{A}^{a}\left(\oplus \mathcal{A}^{b},\right. \\
\mathrm{S} \in m^{a} \text { (1) } m^{b}\left(a^{\alpha}\right) .
\end{array}\right.
\end{array}\right.
$$

Operator $\succ: \quad \Lambda^{a} \succ \Lambda^{b}$ is defined as a quintuplet $\left(U^{a} \cup U^{b}, \tau^{a} \cup \tau^{b}, p^{a} \cup p^{b}, \mathcal{A}^{a} \succ \mathcal{A}^{b}, m^{a} \succ m^{b}\right)$. Operator $\succ$ is purely propositional and has no temporal context. Thus holds the definition $m^{a} \succ m^{b}\left(\mathcal{A}^{a} \succ \mathcal{A}^{b}\right)=\{\mathrm{S}\}$. $\mathcal{A}^{a} \succ \mathcal{A}^{b}$ will combine variable blocks which have the same temporal label as the line containing them. Define $\delta$ by $\delta_{k l}^{\alpha}=0$ if $l \neq k, \delta_{k k}^{a}=-1$ and $\delta_{k k}^{b}=1$. Function $a^{a} \succ_{k} a^{b}$ is stated as follows:

$$
\left\{\begin{array}{l}
\forall \Upsilon \in U^{a} \cup U^{b}, a^{a} \succ_{k} a^{b}(\Upsilon)=\sum_{U^{a} \ni \Upsilon} \delta_{k, \Upsilon^{a}(\Upsilon)}^{\alpha} a^{\alpha}(\Upsilon), \\
a^{a} \succ_{k} a^{b}(1)=0 .
\end{array}\right.
$$

Set $\mathcal{A}^{a} \succ \mathcal{A}^{b}$ is then defined this way:

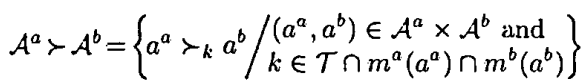

Operator $\succ$ establish some conditional dependency between two LSs. Operator $\otimes$ parallelizes two LSs or make a logical $A N D$ between two LSs. Operator $\oplus$ merges two LSs into a unique, but multi-modal, resource type. Operators $\otimes$ and $(B)$ are associative and commutative. The scalar product is left-distributive w.r.t. $\oplus$ and $\otimes$. It is also right-distributive w.r.t. $\Theta$, see (19). Operator $(1)$ is distributive w.r.t. $($ : the symbols used for the operators may be confusing here...Properties are summed up:

$$
\left\{\begin{array}{l}
\lambda\left(\Lambda^{a} \oplus \Lambda^{b}\right)=\left(\lambda \Lambda^{a}\right) \oplus\left(\lambda \Lambda^{b}\right), \\
\lambda\left(\Lambda^{a} \otimes \Lambda^{b}\right)=\left(\lambda \Lambda^{a}\right) \otimes\left(\lambda \Lambda^{b}\right), \\
(\lambda+\mu) \Lambda^{a}=\left(\lambda \Lambda^{a}\right) \oplus\left(\mu \Lambda^{a}\right), \\
\left(\Lambda^{a} \otimes \Lambda^{b}\right) \oplus \Lambda^{c}=\left(\Lambda^{a}\left(1 \Lambda^{c}\right) \otimes\left(\Lambda^{b} \oplus \Lambda^{c}\right) .\right.
\end{array}\right.
$$

\subsection{Some typical applications}

Multi-mode scheduling: Resources are used, which operate in one of the modes $a$ and $b$, with proper LS $\Lambda^{a}$ and $\Lambda^{b}$. The objective is to optimize the choice between the operating modes so as to minimize $P_{n d}$. It is done by $\Lambda^{a} \oplus \Lambda^{b}$, which concatenates the constraints of $\Lambda^{a}$ and of $\Lambda^{b}$. Refer to 2.3 for example.

Multi-resource scheduling: Resources $a$ and $b$, with proper LS $\Lambda^{a}$ and $\Lambda^{b}$, are given. They are independent, that is $U^{a} \cap U^{b}=\emptyset$. We want $a$ and $b$ to be parallelized, ie resources $a$ can run independently and in same period as resources $b$. The objective is then to allocate $a$ and $b$ together so as to minimize $P_{n d}$. It is simply done by $\Lambda^{a} \otimes \Lambda^{b}$, since constraint $\mathcal{A}^{a} \cup \mathcal{A}^{b}$ combine simultaneously constraints $\mathcal{A}^{a}$ and $\mathcal{A}^{b}$.

Resource dependency: Typically, suppose that resource $a$ can work only when resource $b$ is used. Then, the conditional LS $\Lambda^{b} \succ \Lambda^{a}$ has to be glued by $\otimes$-means to the main LS. Refer to 2.3 for example. 
$\left(\Lambda \otimes \Lambda^{a}\right) \oplus\left(\Lambda \otimes \Lambda^{b}\right)$ and $\Lambda \otimes\left(\Lambda^{a} \oplus \Lambda^{b}\right)$ : These two examples are really different. In first case, resources could be used in mode 1 , with parallelized resources $\Lambda$ and $\Lambda^{a}$, and in mode 2 , with parallelized resources $\Lambda$ and $\Lambda^{b}$. Nevertheless, resources $\Lambda$ released in mode 1 cannot be used directly in mode 2 without releasing resources $\Lambda^{a}$. Parallelized resources have to be combined before jumping from one mode to another. In second case, $\Lambda$ is parallelized with resources working in mode $\Lambda^{a}$ and $\Lambda^{b} . \Lambda$ is used independently to $\Lambda^{a}$ and $\Lambda^{b}$.

\subsection{Practical examples}

Three examples are given here in order to clarify, if needed, the abstract formalism of ICLS. For didactic reason, the constraint of an ICLS $\Lambda^{\alpha}$ will be represented by matrix form $A^{\alpha} \phi^{\alpha} \leq \psi^{\alpha}$. First, we consider independent linear LSs $\Lambda^{a}=\Lambda^{R \infty}$ and $\Lambda^{b}=\Lambda^{R}\left(\frac{1}{4}, \frac{1}{2}\right)$ defined on 4 periods. According to the definition, constraints for $\Lambda^{a}$ and $\Lambda^{b}$ are respectively:

$$
\left(\begin{array}{llll}
1 & 0 & 0 & 0 \\
1 & 1 & 0 & 0 \\
1 & 1 & 1 & 0 \\
1 & 1 & 1 & 1
\end{array}\right) \phi^{a} \leq\left(\begin{array}{l}
0 \\
0 \\
0 \\
0
\end{array}\right) \text { and }\left(\begin{array}{cccc}
1 & 0 & 0 & 0 \\
\frac{3}{4} & 1 & 0 & 0 \\
\frac{1}{4} & \frac{3}{4} & 1 & 0 \\
\frac{1}{4} & \frac{1}{4} & \frac{3}{4} & 1
\end{array}\right) \phi^{b} \leq\left(\begin{array}{l}
0 \\
0 \\
0 \\
0
\end{array}\right)
$$

Define a Multi-resource scheduling of $a$ and $b$ with respective amount of resources 7 and 5 . This problem reverts to $\left(\Lambda^{a}(b-7) \otimes\left(\Lambda^{b}(D)-5\right)\right.$, yielding constraint:

$$
\left(\begin{array}{llllllll}
1 & 0 & 0 & 0 & 0 & 0 & 0 & 0 \\
1 & 1 & 0 & 0 & 0 & 0 & 0 & 0 \\
1 & 1 & 1 & 0 & 0 & 0 & 0 & 0 \\
1 & 1 & 1 & 1 & 0 & 0 & 0 & 0 \\
0 & 0 & 0 & 0 & 1 & 0 & 0 & 0 \\
0 & 0 & 0 & 0 & \frac{3}{4} & 1 & 0 & 0 \\
0 & 0 & 0 & 0 & \frac{1}{4} & \frac{3}{4} & 1 & 0 \\
0 & 0 & 0 & 0 & \frac{1}{4} & \frac{1}{4} & \frac{3}{4} & 1
\end{array}\right)\left(\begin{array}{c}
\phi^{a} \\
\phi^{b}
\end{array}\right) \leq\left(\begin{array}{l}
7 \\
7 \\
7 \\
7 \\
5 \\
5 \\
5 \\
5
\end{array}\right) .
$$

Assume now that resources can run in mode $a$ or in mode $b$, and that $b$ needs 2 times more resources than $a$. Assume moreover that the total amount of resource is 20 when running in mode $a$. This problem reverts to ICLS $\Lambda^{a} \oplus\left(2 \Lambda^{b}\right) \oplus-20$, thus yielding constraint:

$$
\left(\begin{array}{llllllll}
1 & 0 & 0 & 0 & 2 & 0 & 0 & 0 \\
1 & 1 & 0 & 0 & \frac{3}{2} & 2 & 0 & 0 \\
1 & 1 & 1 & 0 & \frac{1}{2} & \frac{3}{2} & 2 & 0 \\
1 & 1 & 1 & 1 & \frac{1}{2} & \frac{1}{2} & \frac{3}{2} & 2
\end{array}\right)\left(\begin{array}{c}
\phi^{a} \\
\phi^{b}
\end{array}\right) \leq\left(\begin{array}{l}
20 \\
20 \\
20 \\
20
\end{array}\right)
$$

As last example, let consider a conditional problem, where the use of a resource $b$ is conditioned to the use of 2 resources $a$. Let the total amount of resources be 10 for $a$ and 5 for $b$. The suitable ICLS is $\left(\Lambda^{a} \oplus-10\right) \otimes\left(\Lambda^{b} \oplus-5\right) \otimes\left(\Lambda^{a} \succ\left(2 \Lambda^{b}\right)\right)$, yielding:

$$
\left(\begin{array}{cccccccc}
1 & 0 & 0 & 0 & 0 & 0 & 0 & 0 \\
1 & 1 & 0 & 0 & 0 & 0 & 0 & 0 \\
1 & 1 & 1 & 0 & 0 & 0 & 0 & 0 \\
1 & 1 & 1 & 1 & 0 & 0 & 0 & 0 \\
0 & 0 & 0 & 0 & 1 & 0 & 0 & 0 \\
0 & 0 & 0 & 0 & \frac{3}{4} & 1 & 0 & 0 \\
0 & 0 & 0 & 0 & \frac{1}{4} & \frac{3}{4} & 1 & 0 \\
0 & 0 & 0 & 0 & \frac{1}{4} & \frac{1}{4} & \frac{3}{4} & 1 \\
-1 & 0 & 0 & 0 & 2 & 0 & 0 & 0 \\
0 & -1 & 0 & 0 & 0 & 2 & 0 & 0 \\
0 & 0 & -1 & 0 & 0 & 0 & 2 & 0 \\
0 & 0 & 0 & -1 & 0 & 0 & 0 & 2
\end{array}\right)\left(\begin{array}{c}
\phi^{a} \\
\phi^{b}
\end{array}\right) \leq\left(\begin{array}{c}
10 \\
10 \\
10 \\
10 \\
5 \\
5 \\
5 \\
5 \\
0 \\
0 \\
0 \\
0
\end{array}\right)
$$

\section{Numerical resolution}

An algorithm is now given for solving ECLS. The ECLS is approximated by series of Brown's problems, and the optimum of the ECLS is given by the optimum of the nearest approximation. To command the choice of these approximations, a differential method (relative to the intermediate optima) is used.

\subsection{Algorithm}

Let $\Lambda=(U, \tau, p, \mathcal{A}, m)$ be an ECLS, and let $A$ and $\psi$ its constraint matrix and vector. For each $\phi$ verifying:

$$
A \phi=\psi,
$$

we define a Brown-like problem $B(\Lambda, \phi)$, which consist$s$ to minimize $P_{n d}(\varphi)$ given in (10) under constraint (7). Resolution of $B(\Lambda, \phi)$ is done by an algorithm almost identical to Brown's algorithm. It will not be explained here. ECLS $\Lambda$ will be approximated by problems $B(\Lambda, \phi)$, where $\phi$ is pushed by infinitesimal variations $d \phi$ to an optimal value $\phi_{\mathrm{A}}$. Criteria have to be found so as to define valid and optimal choice of $d \phi$, satisfying (20). Consider also $A^{\sim}$ a matrix such that $\operatorname{ker} A=\operatorname{Im}\left(A^{\sim}\right)$ and a vector $\phi_{0}$ such that $A \phi_{0}=\psi$ $\left(\phi_{0}\right.$ will be taken as starting value for $\left.\phi\right)$. Then, for each vector $\phi$ fulfilling (20), there is a vector $\nu$ such that $\phi=\phi_{0}+A \widetilde{ }$. Likewise (20) holds true for all $\phi$ of preceding form. Thus, all valid $d \phi$ verifies:

$$
d \phi=A \tilde{d} \nu
$$

Valid choice of $d \phi$ reduces to a choice of $d \nu$. To find an optimal value $\phi_{\mathrm{\Lambda}}$ for $\phi$, i.e. which minimizes $\min _{\varphi} \mathbf{P}_{n d}(\varphi)$, a choice criterion on $d \nu$, based on the calculus of $d \min _{\varphi} \mathbf{P}_{n d}(\varphi)$, is defined in next section. This calculus depends on the current optimal solution $\varphi$ associated to $\phi$. Hence the following algorithm:

1. Compute $A \widetilde{\gamma}, \phi_{0}$ and initialize $\nu=0, \phi=\phi_{0}$

2. Run Brown's algorithm for $B(\Lambda, \phi)$

3. Compute $d \nu$ with solution obtained in 2 .

4. Update $\nu$ and $\phi$ by $\nu:=\nu+d \nu$ and $\phi:=\phi_{0}+\widetilde{\nu}$

5. Return to 2 until convergence. 


\subsection{Optimal choice of $d \nu$}

Differential behavior of $\min _{\varphi} \mathbf{P}_{n d}(\varphi)$ : For each $\mathcal{U} \in \tau^{-1}(\mathcal{T})$, let $\mathcal{T}_{\mathcal{U}}=\mathcal{T} \backslash \tau(\mathcal{U}), t=\operatorname{card}\left(\mathcal{T}_{\mathcal{U}}\right)$ and:

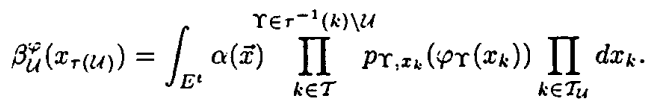

Assume $\varphi$ and $\varphi+d \varphi$ be optimal solutions for $\phi$ and $\phi+d \phi$ respectively. By definition, $d \min _{\varphi} \mathrm{P}_{n d}(\varphi)$ is given by $\mathbf{P}_{n d}(\varphi+d \varphi)-\mathbf{P}_{n d}(\varphi)$. A first order expansion relatively to $d \varphi$ results in linearization (22):

$$
\begin{aligned}
d \min _{\varphi} \mathbf{P}_{n d}(\varphi) & =\sum_{\Upsilon \in \tau^{-1}(\tau)} \int_{E} \beta_{\Upsilon}^{\varphi}(x) \times \\
& \left(p_{\Upsilon, x}\left(\left(\varphi_{\Upsilon}+d \varphi_{\Upsilon}\right)(x)\right)-p_{\Upsilon, x}\left(\varphi_{\Upsilon}(x)\right)\right) d x .
\end{aligned}
$$

This value has to be as negative as possible. Since minimizing a sum reverts to minimizing its members, each $d \varphi_{\Upsilon}$ shall minimize $\int_{E} \beta_{\Upsilon}^{\varphi}(x) p_{\Upsilon, x}\left(\left(\varphi_{\Upsilon}+d \varphi_{\Upsilon}\right)(x)\right) d x$. Such minimization is a kind of 1-period search problem on $\varphi_{\Upsilon}+d \varphi_{\Upsilon}$. Optimality conditions of de Guenin (see (6)) are also found. Since $\varphi$ is optimal too, two de Guenin's conditions are obtained:

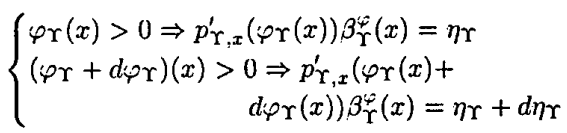

Let $X_{\Upsilon}^{\varphi} \triangleq\left\{x \in E / \varphi_{\Upsilon}(x)>0\right\}, W_{\Upsilon}^{\varphi} \triangleq X_{\Upsilon}^{\varphi+d} \oplus \cap X_{\Upsilon}^{\varphi}$ and $Y_{\Upsilon}^{*} \triangleq X_{\Upsilon}^{\hat{*}}+d_{\hat{\varphi}} \cup X_{\Upsilon}^{*}$. Two cases are considered:

Case $\phi_{\Upsilon}>0$ : Subtraction of equations (23) yields $\forall x \in W_{\Upsilon}, \beta_{\Upsilon}^{*}(x) p_{\Upsilon, x}^{\prime \prime}\left(\varphi_{\Upsilon}(x)\right) d \varphi_{\Upsilon}(x)=d \eta_{\Upsilon}$ and $\forall x \in E \backslash Y_{\Upsilon}, d \varphi_{\Upsilon}(x)=0$. Now, the border integrals $\int_{Y_{\Upsilon} \backslash W_{\Upsilon}} \varphi_{\Upsilon}(x) d x$ and $\int_{Y_{Y} \backslash W_{\Upsilon}}\left(\varphi_{\Upsilon}(x)+d \varphi_{\Upsilon}(x)\right) d x$ are second order infinitesimals, thus yielding :

$$
\int_{X_{\Upsilon}^{\varphi}} \frac{d x}{\beta_{\Upsilon}^{\prime}(x) p_{\Upsilon, x}^{\prime \prime}\left(\varphi_{\Upsilon}(x)\right)} d \eta_{\Upsilon}=\int_{E} d \varphi_{\Upsilon}(x) d x .
$$

Equation (7) differentiates to $\int_{E} d \varphi_{\Upsilon}(x) d x=d \phi_{\Upsilon}$. It comes $d \eta_{\Upsilon}=d \phi_{\Upsilon} / \int_{X_{\Upsilon}^{\varphi}}\left(\beta_{\Upsilon}^{\varphi}(x) p_{\Upsilon, x}^{\prime \prime}\left(\varphi_{\Upsilon}(x)\right)\right)^{-1} d x$. An expression of $d \varphi_{\Upsilon}(x)$ is then given by:

$$
\forall x \in W_{\Upsilon}^{\varphi}, d \varphi_{\Upsilon}(x)=d \phi \Upsilon \frac{\frac{1}{\beta_{\Upsilon}^{\varphi}(x) p_{\Upsilon, x}^{\prime \prime}\left(\varphi_{\Upsilon}(x)\right)}}{\int_{X_{\Upsilon}^{\varphi}} \frac{d x}{\beta_{\Upsilon}^{\varphi}(x) p_{\Upsilon, x}^{\prime \prime}\left(\varphi_{\Upsilon}(x)\right)}} .
$$

Component $\Upsilon$ in sum (22) is thus rewritten:

$$
\begin{gathered}
\int_{E} \beta_{\Upsilon}^{\varphi}(x)\left(p_{\Upsilon, x}\left(\left(\varphi_{\Upsilon}+d \varphi_{\Upsilon}\right)(x)\right)-p_{\Upsilon, x}\left(\varphi_{\Upsilon}(x)\right)\right) d x= \\
d \phi_{\Upsilon} \int_{X_{\Upsilon}^{\varphi}} \frac{p_{\Upsilon, x}^{\prime}\left(\varphi_{\Upsilon}(x)\right)}{p_{\Upsilon, x}^{\prime \prime}\left(\varphi_{\Upsilon}(x)\right)} d x \\
\left(\int_{X_{\Upsilon}^{\varphi}} \frac{d x}{\beta_{\Upsilon}^{\varphi}(x) p_{\Upsilon, x}^{\prime \prime}\left(\varphi_{\Upsilon}(x)\right)}\right.
\end{gathered}
$$

Case $\phi_{\Upsilon}=0: \quad$ Let $\eta_{\Upsilon}^{0}=\max _{x \in E}\left(\beta_{\Upsilon}^{\varphi}(x) p_{\Upsilon, x}^{\prime}(0)\right)$. Equation $\beta_{\Upsilon}^{\prime}(x) \leq\left(\eta_{\Upsilon}^{0}+d \eta_{\Upsilon}\right) / p_{\Upsilon, x}^{\prime}(0) \Rightarrow d \varphi_{\Upsilon}(x)=0$ is derived from de Guenin's conditions. Then, as soon as $d \varphi_{\Upsilon}>0$, property $\beta_{\Upsilon}^{\varphi}(x) p_{\Upsilon, x}^{\prime}(0)=\eta_{\Upsilon}^{0}$ holds. Since $\varphi_{\Upsilon}=0$ (deduced from $\phi_{\Upsilon}=0$ ), equation (25) follows:

$$
\begin{gathered}
\int_{E} \beta_{\Upsilon}^{\varphi}(x)\left(p_{\Upsilon, x}\left(\left(\varphi_{\Upsilon}+d \varphi_{\Upsilon}\right)(x)\right)-p_{\Upsilon, x}\left(\varphi_{\Upsilon}(x)\right)\right) d x= \\
\int_{\beta_{\Upsilon}^{\varphi}(x) \geq \frac{\eta_{\Upsilon}^{0}+d \eta \Upsilon}{p_{\Upsilon, x}(0)}} \beta_{\Upsilon}^{\varphi}(x) p_{\Upsilon, x}^{\prime}(0) d \varphi_{\Upsilon}(x) d x=\eta_{\Upsilon}^{0} d \phi_{\Upsilon} .
\end{gathered}
$$

From properties (24) and (25), equality (22) becomes $d \min _{\varphi} \mathbf{P}_{n d}(\varphi)=\mathrm{V}^{t} d \phi$, with vector $\mathrm{V}$ defined by:

$$
\left\{\begin{array}{c}
\mathrm{V}_{\Upsilon}=\frac{\int_{X_{\Upsilon}^{\varphi}} \frac{p_{\Upsilon, x}^{\prime}\left(\varphi_{\Upsilon}(x)\right)}{p_{\Upsilon, x}^{\prime \prime}\left(\varphi_{\Upsilon}(x)\right)} d x}{\int_{X_{\Upsilon}^{\varphi}} \frac{d x}{\beta_{\Upsilon}^{\varphi}(x) p_{\Upsilon, x}^{\prime \prime}\left(\varphi_{\Upsilon}(x)\right)}} \\
\text { when } \phi_{\Upsilon}>0 \text { and } \Upsilon \in \tau^{-1}(\mathcal{T}) \\
\mathrm{V}_{\Upsilon}=\eta_{\Upsilon}^{0}, \text { when } \phi_{\Upsilon}=0 \text { and } \Upsilon \in \tau^{-1}(\mathcal{T}) \\
\mathrm{V}_{\Upsilon}=0, \text { when } \tau(\Upsilon)=\mathrm{S}
\end{array}\right.
$$

Choice of $d \nu$ : Main result (27) is deduced from (21). $d \nu$ is chosen, as a minimizer of $d \min _{\vartheta} \mathbf{P}_{n d}(\varphi)$.

$$
d \min _{\varphi} P_{n d}(\varphi)=\mathbf{V}^{t} A^{\tau} d \nu
$$

Practically, discretization of $d \nu, \Delta \nu$, results in positivity gaps. Thus, $\Delta \nu$ is given by (28):

$$
\begin{aligned}
& \text { Minimize } \mathrm{V}^{t} A^{\sim} \Delta \nu, \\
& \text { subject to } A^{\sim} \Delta \nu+\phi \geq 0 \text { and }\left\|A^{\sim} \Delta \nu\right\| \leq \Delta t .
\end{aligned}
$$

When \|\|$=\|\|_{\infty},(28)$ is linear and can be straightforwardly solved by means of the Simplex algorithm.

\section{Results}

Our algorithm is rather fast. Computation of $\Delta \nu$ is negligible, compared to Brown's process. Thus, the speed of our algorithm is of the same order than Brown's one. In this section, two examples are presented. They are produced with operators $\otimes$ and $\oplus$ from basic models of resources, and correspond to multiresource and multi-mode scheduling. The space search $E$ is here a square of $30 \times 30$ cells. The detection occurs during $T=7$ periods. The target has a diffusion movement to down and right. Its start position is uniform on the top-left $10 \times 10$ square, centered around the point $(1,1)$. So, the probabilistic prior about the target is $\alpha(\vec{x})=s\left(x_{1}\right) \prod_{k=1}^{6} m\left(x_{k+1}-x_{k}\right)$, where:

$$
\left\{\begin{array}{l}
s(x)=\frac{1}{100} \text { if }(1,1) \leq x \leq(10,10), s(x)=0 \text { else, } \\
m(0,0)=m(3,3)=\frac{3}{14}, m(2,3)=m(3,2)=\frac{2}{14} \\
m(0,3)=m(3,0)=m(1,3)=m(3,1)=\frac{1}{14} \\
m(\mathbf{x})=0 \text { else. }
\end{array}\right.
$$

All non detection function will be exponential, i.e. $p_{\Upsilon, x}(\varphi)=\exp \left(-\omega_{\Upsilon, x} \varphi\right)$. The visibility parameter $\omega_{\Upsilon, x}$ will not depend on the period of detection. 


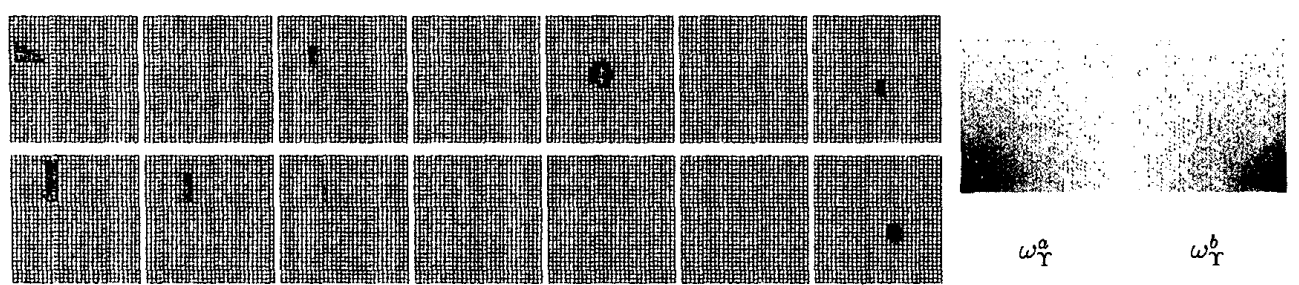

Figure 1: First example: $\Lambda^{a} \otimes \Lambda^{b}$

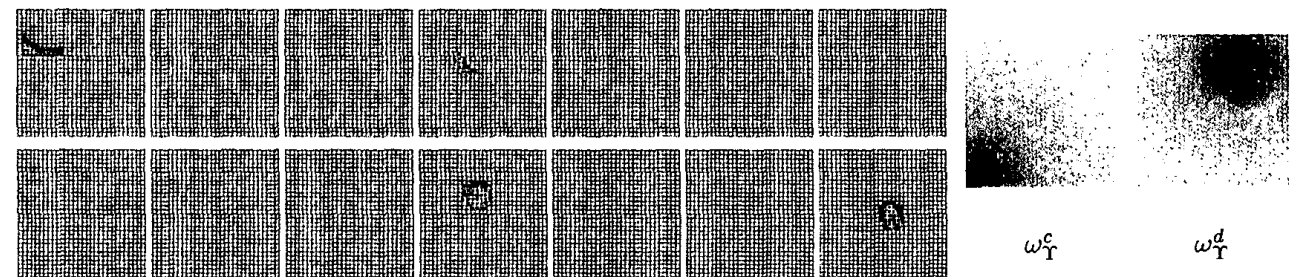

Figure 2: Second example: $\left(2 \Lambda^{c}\right) \oplus \Lambda^{d} \oplus-200$

First example: Two basic resources are used,
which correspond to LS $\Lambda^{a}=\Lambda^{R 4} \oplus-100$ and to LS which correspond to LS $\Lambda^{a}=\Lambda^{R 4} \oplus-100$ and to LS
$\Lambda^{b}=\Lambda^{R \infty} \oplus-200$. LS $\Lambda^{b}$ represents also non renewable resources with amount equal to 200 . Likewise, $\Lambda^{a}$ represent resources renewable after 4 periods and with amount equal to 100 . Non detection functions $p^{a}$ and $p^{b}$ are defined by their visibility parameters $\omega_{\Upsilon, x}^{a}$ and $\omega_{\Upsilon, x}^{b}$. Representation of $\omega_{\Upsilon, x}^{a}$ and $\omega_{\Upsilon, x}^{b}$ are given in figure 1. $p^{a}$ is also stronger down-left, decreasing with the radius, since $p^{b}$ is stronger down-right. Algorith$\mathrm{m}$ has been applied to the resource scheduling of $\Lambda^{a}$ and $\Lambda^{b}$. As already seen, such problem is described by ICLS $\Lambda^{a} \otimes \Lambda^{b}$. Results are viewed in figure 1. For resources $a$, the global amounts of resources, $\phi_{\Upsilon}^{a}$, are $78,0,22,0,78,0,22$, for $\Upsilon$ equals periods 1 to 7 . The spatial repartitions $\varphi_{\Upsilon}^{a}$ are represented in the same order in the upper part of figure 1. For resources $b$, the global amounts of resources are $110,37,6,2,0,0,44$. The spatial repartitions are represented in the lower part of figure 1. Note that the sum of four consecutive global amounts of resources $a$ equals 100 . In the same way, the sum of all global amounts of resources $b$ equals 200 . Those conform to the renew constraints on $a$ and $b$. Since the target is dispersive, it is natural that the most resources are used at first periods. Again, since $a$ renews after 4 periods, resources $a$ appear at period 5. Now, visibility for $b$ is better down-right. and thus some resources are used at period 7. Spatially, resources $a$ are rather placed down-left, since resources $b$ are rather down-right, according to the visibility parameters. Some surrounding occurs at the first periods.

Second example: Two basic resource modes are used, which correspond to LS $\Lambda^{c}=\Lambda^{R 3}$ and to LS $\Lambda^{d}=\Lambda^{R \infty}$. LS $\Lambda^{c}$ represents also a 3 -periods renewable resource mode. Likewise, $\Lambda^{d}$ represents a non renewable resource mode. Functions $p^{c}$ and $p^{d}$ are represented in figure 2. $p^{c}$ is stronger down-left, since $p^{d}$ is stronger near the up-right side. Algorithm has been applied to the mode scheduling of $\Lambda^{c}$ and $\Lambda^{d}$. The resources can also either run like resources $c$ or like resources $d$, but it is assumed that mode $c$ uses two times more resources than mode $d$. Total resource amount is fixed such that it equals 200 , when running in mode $d$ all the time. Such problem is described by ICLS $\left(2 \Lambda^{c}\right) \oplus \Lambda^{d} \oplus-200$. Results are viewed in figure 2. For mode $c$, global amounts of resources are $100,0,0,48,0,0,0$. The local repartitions are represented by the upper part of figure 2 . For mode $d$, global amounts of resources are $0,0,0,104,0,0,96$. The local repartitions are represented by the lower part of figure 2. It appears that it is better to use resources in renewable mode $c$ at the beginning, and in non renewable mode $d$ ( $d$ is more powerful and profitable but destroys resources) at the end. Since 1 resources $c$ is equivalent to 2 resources $d, \frac{200}{2}=100$ resources are used at period 1 in mode $c$. The resources used in mode $c$ renew after 4 periods. That why 48 resources in mode $c$ and $2 \times 52=104$ resources in mode $d$ become available at period 4 . Then 48 resources renew from mode $c$ at period 7 (no renew from mode $d$ ). Thus, at period 7 , only $2 \times 48=96$ resources are eventually available in mode $d$. Spatially, resources $c$ are rather placed down-left, since resources $d$ are rather up-right. A slight surrounding occurs at period 1 .

Third example: This last example involves three kinds of resources, say $e, f$ and $g$. Resources $e$ are 2-periods renewable with amount equal to 115 . Their corresponding LS is $\Lambda^{e}=\Lambda^{R 2} \oplus-115$. Resources $f$ are non renewable with amount equal to 200 . Their corresponding LS is $\Lambda^{f}=\Lambda^{R \infty} \oplus-200$. Resources $g$ are gradually renewable following sequence $\left(\frac{1}{8}, \frac{1}{4}, \frac{1}{8}\right)$. When 1 resource $g$ is used at period $k$, then $\frac{1}{8}$ of this resource is renewed at period $k+1, \frac{1}{4}$ at period $k+2$ and $\frac{1}{8}$ at period $k+3$. So, $\frac{1}{2}$ of the resource is definitively lost. The amount of these resources is 100 . Their corresponding LS is then $\Lambda^{g}=\Lambda^{R}\left(\frac{1}{8}, \frac{1}{4}, \frac{1}{8}\right) \oplus-100$. Resource $e$ will be used as a control resource for the two 

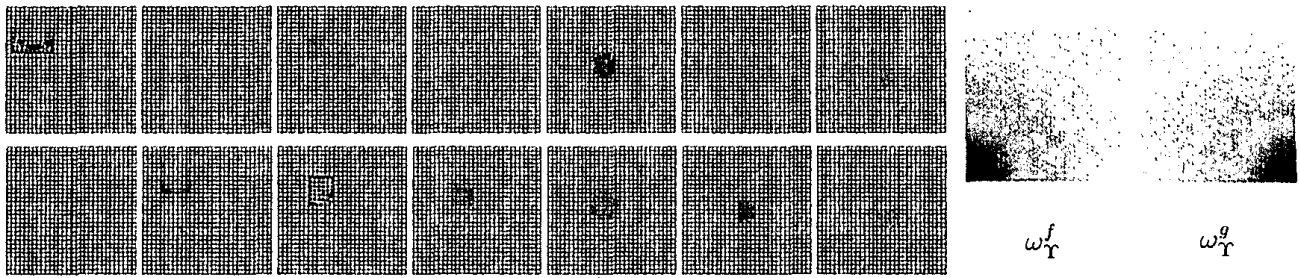

Figure 3: Third example: $\left(\Lambda^{e} \succ\left(\Lambda^{f} \oplus \Lambda^{g}\right)\right) \otimes \Lambda^{e} \otimes \Lambda^{f} \otimes \Lambda^{g}$

other, that means in this example that the use of either 1 resource $f$ or 1 resource $g$ requires the use of 1 resource $e$. To be more precise, it requires 2 resources $e$, when using at same time 1 resource $f$ and 1 resource g. Such condition is stated by LS $\Lambda^{e} \succ\left(\Lambda^{f} \oplus \Lambda^{g}\right)$. Otherwise, all resources are assumed to run independently, and the whole problem is represented by mean of ICLS $\left(\Lambda^{e} \succ\left(\Lambda^{f} \oplus \Lambda^{g}\right)\right) \otimes \Lambda^{e} \otimes \Lambda^{f} \otimes \Lambda^{g}$. Functions $p^{f}$ and $p^{g}$ are represented in figure 3. $p^{f}$ is stronger down-left, since $p^{g}$ is stronger down-right. Since resource $e$ is only used for control, it accomplish no detection, so that $p^{e}=0$. Results are viewed in figure 3. For mode $e$, global amounts of resources are $100,15,100,15,97,18,25$. The local repartition$\mathrm{s}$ are given by adding up the local repartition of $f$ and $g$. For mode $f$, global amounts of resources are $100,0,13,0,72,0,15$. The local repartitions are represented by the upper part of figure 3 . For mode $g$, global amounts of resources are $0,15,87,15,25,18,10$. The local repartitions are represented by the lower part of figure 3. The reader could verify that the constraints on resources are actually checked. It is also uneasy to make comments on such complex example. Nevertheless, it is noteworthy that the resources $f$ and $g$ are not put mainly on the fist period, where target dispersion begins. The reason is the lack of control resource $e$, which is limited by 115 . Thus, the use of resources are more spread between the search periods. Surrounding aspect are stronger in this conditioned example.

\section{Conclusion and perspectives}

Our aim was to solve the problem of spatial and temporal resources sharing. To handle such problem, the algorithm of Brown-de Guenin has been really improved. Our algorithm is robust and fast, similarly to Brown's one. Moreover, in addition with the linear operational formalism we developed, it seems sufficiently general to handle problems of sensor managements, involving various types of sensors and operating modes. The underlying semantic of our model even permits conditional aspects in resource allocation problems and to introduce controls in lateral splitting. These points have been considered in a general setting. For specific applications, more work has to be done; however our approach seems sufficiently open.

\section{References}

[1] S.J. Benkovski, M.G. Monticino and J.R. WeIsinger, A Survey of the Search Theory Literature. Naval Research Logistics, vol.-38, pp. 469491, 1991.

[2] L.D. STone, Theory of Optimal Search, 2-nd ed. . Operations Research Society of America, Arlington, VA, 1989.

[3] K. IIDA, Studies on the Optimal Search Plan. Lecture Notes in Statistics, vol. 70, Springer-Verlag, 1992.

[4] J. de GuenIN, Optimum Distribution of Effort: an Extension of the Koopman Basic Theory. Operations Research 9, pp 1-7, 1961.

[5] S.S. BRown, Optimal Search for a Moving Target in Discrete Time and Space. Operations Research 28, pp 1275-1289, 1980.

[6] A.R. WASHBURN, Search for a moving Target: The FAB algorithm. Operations Research 31, pp 739-751, 1983.

[7] T. Ibaraki and N. Katolr, Resource Allocation Problems: Algorithmic Approaches. MIT Press, 1988.

[8] D.H. Wagner, W.C. Mylander and T.J. Sanders edts, Naval Operations Analysis (3-rd edition), Chapt. 5. Naval Institute Press, Annapolis, MD, 1999. MIT Press, 1988. 